\title{
EDITORIAL
}

\section{The present and future of DAO}

\author{
Otto Kinne ${ }^{1, *}$, Sven Klimpel ${ }^{2, * *}$, Alex D. Hyatt ${ }^{3, * * *}$ \\ ${ }^{1}$ International Ecology Institute, Nordbünte $23(+5,28,30), 21385$ Oldendorf/Luhe, Germany \\ ${ }^{2}$ Institute of Zoomorphology, Cell Biology and Parasitology, Heinrich Heine University, Universitätsstr. 1, \\ 40225 Düsseldorf, Germany \\ ${ }^{3}$ Electron Microscopy Group, Australian Animal Health Laboratory, CSIRO, Livestock Industries, Private Bag 24, Geelong, \\ Victoria 3220, Australia
}

A major part of the world's human population lives close to aquatic environments (seas, oceans, lakes and rivers). Sustainable use of these environments and their aquatic resources is therefore one of the greatest challenges for human existence.

In this context, diseases of aquatic organisms, including aquatic parasitology, constitute increasingly important fields of study. Diseases and parasites affect all aquatic life. Parasites can infest all organs, and thus may lead to mortality, impaired reproduction, or reduced growth. Such a great range of negative consequences may not only affect individual aquatic organisms, but also populations and ecosystems.

Human activities such as overfishing and environmental degradation threaten many fish stocks and also impair fisheries production in terms of rearing aquatic organisms (semi-extensive or intensive aquaculture). Intensive cultivation tends to augment infections (epidemics) by viruses, bacteria and fungi, as well as by protozoan and metazoan parasites. Frequently, such pathogens are introduced via seedlings and fry, or are transmitted by wild stocks living near culture facilities.

Lafferty et al. (2004) reviewed the effects of anthropogenic stressors on the prevalence of marine diseases: climate warming may cause thermal stress; coastal development leads to habitat destruction or degradation; introduced species may act as disease vectors; fishing activities tend to reduce the abundance of common species, thus promoting non-target species; runoff and discharges contain nutrients and pathogens along with fertilizers, pesticides, antibiotics, antihelminthics, and herbicides. Both host species and disease agents are affected by these stressors.

Inter Research (IR), the publisher of Diseases of Aquatic Organisms (DAO), has expanded the scope of the journal to explicitly include parasitology, and has appointed 2 new Editors-in-Chief: Sven Klimpel and Alex Hyatt. The editorial board of DAO will be expanded in the near future to include Editors covering general aspects of diseases (until now handled by Prof. Otto Kinne, founder and for many years Managing Editor of DAO), as well as effects of global warming and environmental change.

Otto Kinne and the members of the Editorial Board have built DAO into the world's leading journal on aquatic diseases. The new Editors-in-Chief strive to maintain this legacy. DAO invites high-quality research papers on field and laboratory investigations. Submitted manuscripts will be handled immediately and efficiently. They are pre-screened by the Editorsin-Chief and by Editors. Although all manuscripts must be submitted to the journal's editorial office (www.intres.com/journals/dao/), authors are encouraged to identify their preference of the Editor for handling the peer review of their submission.

IR solicits 3 to 4 reviewers for every manuscript and thus aims at the highest possible standard of quality control. In cases of poor quality or topical unsuitability, Editors may return manuscripts without review. The editorial policies and procedures of DAO (www. int-res.com/journals/dao/information/) conform to the recommendations of the Council of Science Editors (CSE 2006; see also Kinne 1988, 2002, 2003). The Editorial Board is dedicated to ensuring that DAO continues to publish the best science on the diseases of aquatic organisms.

\section{LITERATURE CITED}

Council of Science Editors (2006) CSE's white paper on promoting integrity in scientific journal publications (available at: www.councilscienceeditors.org/editorial_policies/ white_paper.cfm)

Kinne O (1988) The scientific process - its links, functions and problems. Naturwissenschaften 75:275-279

Kinne O (2002) Importance and organisation of direct postpublication critique. Mar Ecol Prog Ser 228:1

Kinne O (2003) Quality control in science: recruiting, protecting and controlling the controllers. Clim Res 25:1

Lafferty KD, Porter JW, Ford SE (2004) Are diseases increasing in the ocean? Annu Rev Ecol Evol Syst 35:31-54

\footnotetext{
*Email: kinne@int-res.com

**Email: sven.klimpel@uni-duesseldorf.de

${ }^{* * *}$ Email: alex.hyatt@csiro.au
}

(C) Inter-Research $2008 \cdot$ www.int-res.com 